\title{
Problem solving within a verbal response hierarchy'
}

CARL P. DUNCAN

NORTHWESTERN UNIVERSITY

IIpon being given a stimulus word, Ss were given three guesses to discover the strongest or the second strongest associate of the word. As expected from response hierarchy approach to problem solving, improvement over successive guesses was greater for the weaker associate.

Response hierarchy approach to problem solving (Maltzman, 1955) would suggest that if the solution response is high in the hierarchy of responses elicited by the problem stimulus, S's first guess at the solution response will more likely be correct than if the solution is lower in the hierarchy. But if the first guess is incorrect and $\mathrm{S}$ is allowed to make additional guesses, such subsequent guesses should be of responses lower in the hierarchy. Therefore, measured by the first guess alone, performance should be better if the response to be discovered is high in the hierarchy than if it is low. However, on subsequent guesses, this difference should be reduced; there should be a relatively greater gain in performance on the low-hierarchy response. In addition to testing this notion, the present experiment includes a measure of directionality of associations in a guessing situation.

Method

The materials were 20 Kent-Rosanoff stimulus words, plus the first (strongest) and the second associate for each stimulus, all taken from the Russell \& Jenkins norms (1954). For some of the items, the strength of the first associate is relatively high, for others, relatively low.

E said to S, "I want you to try to guess a word that I am thinking of. I will give you a word that is associated with the word I'm thinking of, and then you try to guess the word. I will let you have three guesses. All right, the word that is associated with the word I'm thinking of is..."

For all Ss in both Group 1 and Group 2, E gave S the Kent-Rosanoff stimulus word for all 20 items. For Group $1 \mathrm{Ss}$, the first associate was the word to be guessed. In Group 2, the second associate was correct. If $\mathrm{S}$ got the correct word before taking ihree guesses, $E$ went on to the next item. If S did not get the correct word in three guesses, he was not told the word. For every item, E said, "The associated wurd is...", or, "The associate is..." The 20 items were arranged in five different random orders which were assigned to Ss in turn.

Group 1 and Group 2 each contained 50 Ss. An additional group of $50 \mathrm{Ss}$, Group $\mathrm{S}$, was given the first associate for each item and allowed three attempts to guess the stimulus word. Comparison of Group
$\mathrm{S}$ with Group 1 provides information on the role of directionality of associations in a response discovery situation.

\section{Results and Discussion}

Since the expectation was that Groups 1 and 2 would show differing patterns of performance from the first guess to subsequent guesses, two scores were obtained for each $\mathrm{S}$. One was the number correct on the first guess. The other was the sum of the number correct on the second and third guesses combined. For Group 1, the mean correct on the first guess was 8.82 , the mean on guesses two and three was 4.20. For Group 2 , the mean on guess one was 2.94, the mean on guesses two and three was 4.62 . Thus, as expected, relative to the first guess, the pertormance of Group 2 improves on later guesses. The interaction of groups $\mathbf{x}$ guesses suggested by the four means is highly significant, $F$ was 142.9, p .001 with 1 and 98 df. As can be seen, even though Group 1 had a much higher mean than Group 2 on the first guess, Group 2 did slightly better than Group 1 on later guesses.

When $\mathrm{S}$ is asked to guess a word that is associated with some given word, it is not clear whether $S$ treats the given word as a stimulus which elicits an associate that he is to guess, or whether he treats the given word as an associate whose stimulus he must guess. Comparison of Group 1 (given the Kent-Rosanoff stimulus word, guess the first associate) with Group $\mathrm{S}$ (given the first associate, guess the stimulus) provides evidence on this point. Over all 20 items, these groups did not differ. Mean correct first guesses were 8.82 for Group 1, 9.24 for Group S. Mean total correct guesses (after three guesses) were 13.02 for Group 1, 11.98 for Group S. Neither of these mean differences is significant. Examination of individual items revealed that for many of them, the stimulus and its strongest associate were strongly and equally associated in both directions, measured by number correct over all three guesses. These were usually opposites such as sweet-sour. Others were mediumly and equally associated, e.g., wish-want. Strong unidirectional associates were river-water, and stomachfood; the stimulus (first word) elicited the associate but rarely vice versa. In two items, whistle-stop, and ciizen-U.S.A., there was little evidence, by the guessing method used in this study, of association in either direction.

The results suggest that if $S$ is trying to discover the solution response to a problem, his successive guesses tend to follow a response hierarchy pattern. Several consequences follow. If the solution response 
is very high in the hierarchy, many, though not all, first guesses will be correct. Subsequent guesses are not likely to increase greatly the total number of correct responses since such guesses should tend to be of responses lower in the hierarchy, which is not conducive to discovering a high-hierarchy response. However, if the solution response is a low-hierarchy response, first guesses should infrequently be correct, but performance should improve, relatively, on later guesses.

\section{References}

Maltzman, 1. Thinking: From a behavioristic point of view. Psychol. Rev., 1955, 62, 275-286.

Russell, W. A., \& Jenkins, J. J. The complete Minnesota norms for responses to 100 words from the Kent-Rosanoff Word Association Test. Office of Naval Research, Tech. Report 11, 1954.

\section{Note}

1. This study was supported by grant HD00901 from the Institute of Child Health and Human Development, National Institutes of Health, United States Public Health Service. Thanks are due Gordon Wood, Judith Pagel, and Linda Shaffer. 